\title{
Splenic Artery Embolization in Blunt Trauma
}

\author{
${ }^{1}$ Department of Radiology, University of Colorado, Denver Anschutz \\ Medical Campus, Aurora, Colorado \\ Semin Intervent Radiol 2012;29:147-149
}

Brian F. Imbrogno, M.D. ${ }^{1}$ Charles E. Ray, Jr., M.D., Ph.D., F.S.I.R. ${ }^{1}$

In prior decades, splenic arterial embolization (SAE) was used increasingly in the setting of nonoperative management of blunt splenic trauma. Recently, however, multiple studies have questioned the efficacy of SAE in the blunt trauma population; at the very least, the clinical indications for SAE appear to be changing. ${ }^{1}$ At many institutions, however, including ours, SAE remains a mainstay in the treatment of patients suffering blunt trauma to the spleen.

Although there is evidence that in certain patient populations SAE improves outcome, the superiority of a specific technique (proximal embolization, distal embolization, or a combination of both) is still debated. This article briefly reviews indications, general techniques, and complications of SAE; specific attention is paid to our preferred technique for SAE, with an emphasis on the distinction between proximal and distal embolization.

\section{Indications}

At our institution, hemodynamically stable patients are evaluated with contrast-enhanced computed tomography (CT). The American Academy of Surgery and Trauma Organ Injury Scale (AAST-OIS) is a widely accepted grading scale for solid organ injuries. ${ }^{2}$ There is some debate as to the reliability of CT to characterize splenic injury accurately, and some evidence suggests angiographic findings correlate better with patient outcomes. ${ }^{3}$ However, angiography has its own risks, as well as being less timely and available; therefore sending all hemodynamically stable blunt splenic trauma patients to angiography would be extreme. Treatment decisions currently are being made on the appearance of the spleen on CT, as well as various clinical factors.

As a general rule, patients who are hemodynamically unstable are managed surgically. On rare occasions, individuals who are borderline hemodynamically unstable with documented isolated splenic injuries undergo SAE rather than an operative procedure. In the hemodynamically stable patient, SAE may be utilized depending on several factors, many of which vary from one institution to another. Although highly variable, the need for SAE is generally based on a combination of clinical condition (hemodynamic status, on-
Address for correspondence and reprint requests Charles E. Ray, Jr., M.D., Ph.D., F.S.I.R., University of Colorado Anschutz Medical Campus, Stop L954, 12401 E. 17th Avenue, Room 526, Aurora, CO, 80045 (e-mail: Charles.ray@ucdenver.edu).

going bleeding as evidenced by decreasing hematocrit level) and imaging findings (contrast extravasation, grade of injury, direct evidence of vascular injury such as pseudoaneurysm/ arteriovenous fistula $[A V F])$. At our institution, we currently reserve SAE for individuals who are hemodynamically stable and who meet one of three criteria: those with active contrast extravasation on CT, those with severe injury (grade 3 or higher) and decreasing hematocrit levels, and those with visualized vascular injury and decreasing hematocrit levels. These criteria have admittedly become more strict over time, and as a result we are performing far fewer splenic embolization procedures now than we did 5 to 10 years ago.

Once the decision has been made to proceed with angiography, the next decision is whether or not to embolize based on angiographic findings. Active bleeding, pseudoaneurysm, AVF, or other vascular injury seen at angiography are sufficient findings to proceed with SAE. However, in patients with abnormal CT and normal angiography, the decision to embolize becomes less clear. Although some authors recommend embolization based empirically on the CT findings (e.g., grade III to $\mathrm{V}$ injury without angiographic evidence of vascular injury), ${ }^{4}$ our practice is to base the embolization more on the angiographic findings and clinical evidence for active hemorrhage.

\section{Technique}

General angiographic technique is typically used. Access is obtained from a femoral approach with a micropuncture kit, after which the celiac artery is selected with a curved or reversed curve catheter (Cobra, Simmons 1 or 2, respectively; Cook Medical, Inc., Bloomington, IN). Prior to selecting the splenic artery, a celiac arteriogram should be performed with particular attention not only to the splenic artery but the left gastric artery as well because it can be an important collateral feeder to the spleen. The main splenic artery is then catheterized and a splenic arteriogram performed.

If a distal embolization procedure is to be performed, a microcatheter and wire combination (e.g., Renegade STS catheter and Fathom 0.016-inch wire; Boston Scientific, Natick, MA) are placed as distally and near to the injured vessel as possible. We typically use a microcatheter with a
Copyright @ 2012 by Thieme Medical Publishers, Inc., 333 Seventh Avenue, New York, NY 10001, USA. Tel: +1(212) 584-4662.
DOI http://dx.doi.org/ $10.1055 / \mathrm{s}-0032-1312577$. ISSN 0739-9529. 
standard rather than high-flow lumen, due to the fact that a pushable coil may be used during the embolization procedure. High-flow lumen microcatheters may allow a coil to partially coil within the lumen, causing blockage of the microcatheter; they may also allow for premature deployment inside the catheter if detachable coils with mechanical detachment mechanisms are used (e.g., Interlock, Boston Scientific). Because the spleen is generally considered to have an end-organ vascular supply, it is important to place the microcatheter as distally as possible before embolization to preserve as much native supply to the spleen as possible. Once the microcatheter is properly positioned, embolization can be performed with a variety of agents: Gelfoam (Upjohn, Inc., Kalamazoo, MI), particles, or coils can be used in isolation or together. We tend to use either a Gelfoam slurry or medium-size particles ( 300-500 $\mu \mathrm{m})$ (-Fig. 1).

For a proximal embolization procedure, the goal of the embolization is to simply occlude the main splenic artery to decrease the perfusion pressure of the spleen while allowing perfusion of the splenic tissue via collateral pathways. Important collateral pathways include short gastric arteries, pancreatic arteries, gastroepiploic arteries, and splenic capsular arteries. To preserve the pancreatic collateral pathway,
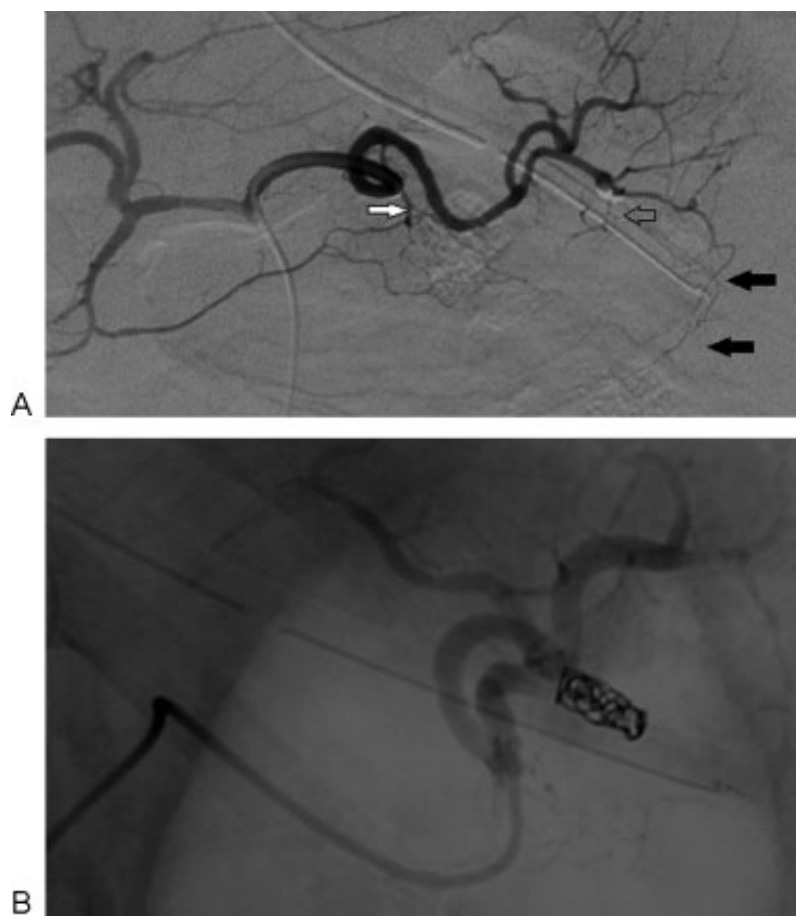

Figure 1 Distal splenic embolization in a 30-year-old man with lower pole grade $V$ splenic injury. (A) Splenic arteriogram, anteroposterior (AP) projection, midarterial phase, demonstrating attenuation of intrasplenic branches supplying the inferior pole of the spleen, as well as mass effect by the intrasplenic hematoma evidence by splaying of the inferior pole branches (black arrows). Note the dorsal pancreatic artery (white arrow) and arterial pancreatica magna (open arrow), both of which provide important collateral pathways in the setting of proximal splenic artery embolizations. (B) Splenic arteriogram, AP projection, midarterial phase, after selective coil embolization of the lower pole splenic arterial branch. This is considered a distal splenic artery embolization because the embolization occurred beyond the splenic hilum and distal to any major potential collateral pathways. we specifically place our coil pack just distal to the dorsal pancreatic artery origin (typically in the proximal third of the splenic artery). This allows for perfusion of the spleen via the dorsal pancreatic-transverse pancreatic-arteria pancreatica magna-splenic artery pathway; it is not at all uncommon to see that pathway develop immediately after the embolization procedure (-Fig. 2 ).

Although evidence is limited, a recent meta-analysis suggests no significant difference between proximal and distal embolization techniques when comparing major complications requiring splenectomy (infection, infarction, and/or rebleeding). ${ }^{5}$ However when comparing nonoperative complications between individuals undergoing proximal versus distal embolization, the latter was found to have a statistically significant higher rate of splenic infarction not requiring splenectomy. A proposed theory to explain this is that proximal embolization decreases the pressure head sufficiently to control bleeding while allowing collateral vessels to perfuse the spleen, resulting in a lower incidence of ischemia/infarction.

Another argument for proximal over distal embolization is efficiency and shorter duration of the procedure. This may be important for a variety of reasons. First, although these patients are generally hemodynamically stable, in the setting

A
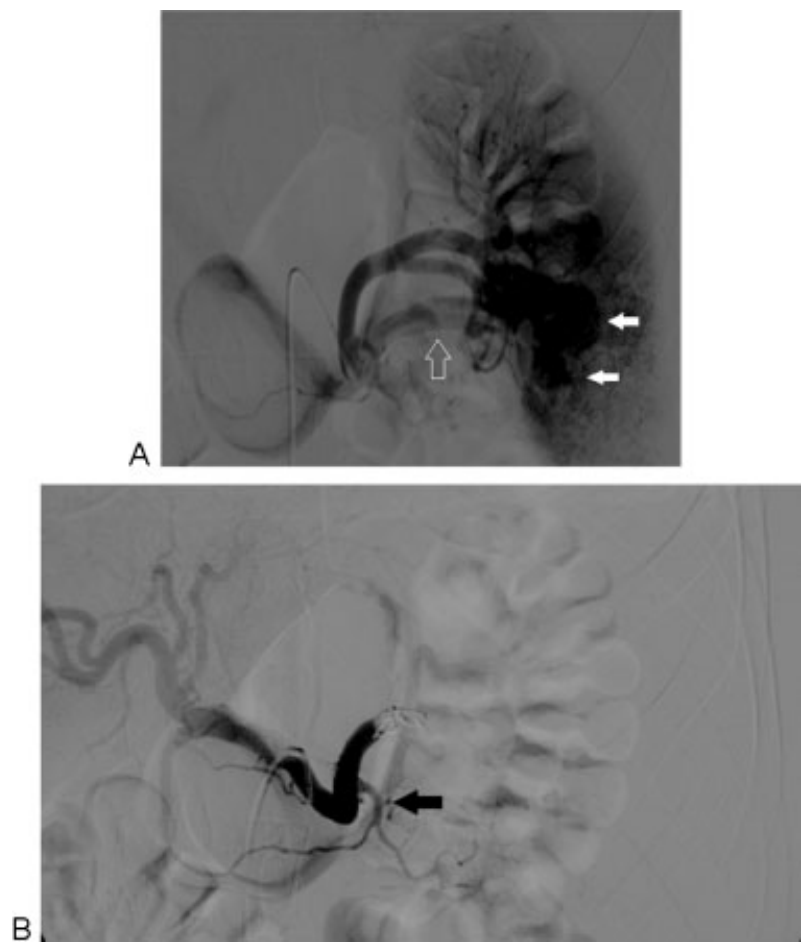

Figure 2 Proximal splenic embolization in a 42-year-old man after blunt traumatic injury to his spleen. A contrast-enhanced computed tomography scan (not shown) demonstrated a hypervascular enhancing structure just distal to the splenic hilum. (A) Splenic arteriogram, anteroposterior (AP) projection, midarterial phase,

demonstrating hypervascular structure within the splenic parenchyma (white arrows) and an early draining splenic vein (open arrow).

(B) Splenic arteriogram, AP projection, midarterial phase, following proximal coil embolization of the main splenic artery. Note the coils are placed just distal to the dorsal pancreatic artery (arrow). 
of active hemorrhage hemodynamic status can change quickly; selecting small splenic arterial branches can be arduous and time consuming. Second, these patients frequently have multiple injuries and medical needs, and decreasing the amount of time spent in the angiography suite may facilitate access to other requisite treatments. Third, many of these trauma patients are relatively young, and fluoroscopy time/ radiation dose should be a concern. Although there has been no study comparing overall procedure and fluoroscopy times in proximal versus distal embolizations, it seems somewhat intuitive to the us that the latter would require more of both.

One potential downside to proximal embolization is the inability to treat rebleeding distal to the embolization site. This is normally not an issue in the trauma patient population in which one embolization is almost always sufficient. But it can become a more significant consideration in populations with comorbidities such as portal hypertension, hypersplenism, or other predisposition to recurrent hemorrhage.

\section{Conclusion}

In our experience and based on the current literature, proximal embolization has been shown to effectively control bleeding with potentially fewer complications and at least equal efficiency than distal/selective embolization. For these reasons, as well as the shorter procedure time and likely lower radiation dose, we prefer to perform proximal embolization in trauma patients in nearly all circumstances.

\section{References}

1 Smith HE, Biffl WL, Majercik SD, Jednacz J, Lambiase R, Cioffi WG. Splenic artery embolization: Have we gone too far? J Trauma 2006;61(3):541-544; discussion 545-546

2 Tinkoff G, Esposito TJ, Reed J, et al. American Association for the Surgery of Trauma Organ Injury Scale I: spleen, liver, and kidney, validation based on the National Trauma Data Bank. J Am Coll Surg 2008;207(5):646-655

3 Shanmuganathan K, Mirvis SE, Boyd-Kranis R, Takada T, Scalea TM. Nonsurgical management of blunt splenic injury: use of CT criteria to select patients for splenic arteriography and potential endovascular therapy. Radiology 2000;217(1):75-82

4 Bessoud B, Denys A, Calmes JM, et al. Nonoperative management of traumatic splenic injuries: is there a role for proximal splenic artery embolization? AJR Am J Roentgenol 2006;186(3): 779-785

5 Schnüriger B, Inaba K, Konstantinidis A, Lustenberger T, Chan LS, Demetriades D. Outcomes of proximal versus distal splenic artery embolization after trauma: a systematic review and meta-analysis. J Trauma 2011;70:242-260 